ORIGINAL ARTICLE

\title{
Cough plate versus cough swab in patients with cystic fibrosis; a pilot study
}

\author{
S Maiya, M Desai, A Baruah, P Weller, J R Clarke, J Gray
}

Arch Dis Child 2004;89:577-579. doi: 10.1136/adc.2003.037481

See end of article for authors' affiliations

.....................

Correspondence to:

Dr M Desai, Department of

Respiratory Medicine, Birmingham Children's

Hospital, Steelhouse Lane,

Birmingham B4 6NH, UK; maya.desai@bch.nhs.uk

Accepted 21 October 2003

\begin{abstract}
Background: Accurate identification of lower respiratory tract pathogens is important in the management of cystic fibrosis patients.

Aim: To evaluate the cough plate as an alternative method of obtaining respiratory samples for microbiological culture.

Methods and Results: Using sputum culture as the "gold standard", the cough plate method identified significantly more positive cases than either dry or moistened cough swabs. Of 20 patients who had a positive sputum culture, 16 had a positive culture on cough plate compared to seven positive cultures each on moistened swab and on dry swab respectively.

Conclusions: In this study cough plates were more sensitive than cough swabs in isolating respiratory pathogens in sputum producers. As patients prefer it, the cough plate merits further evaluation, particularly in non-sputum producers.
\end{abstract}

l: n the 1930s, when cystic fibrosis (CF) was first described most affected children died in the first few years of life.

Over the past decades survival of CF patients has increased, mainly as a result of aggressive antibiotic policies and improved nutrition. Chronic colonisation of the respiratory tract with bacterial pathogens such as Pseudomonas aeruginosa is strongly associated with progression of pulmonary disease. ${ }^{12}$ Thus accurate identification of lower respiratory tract pathogens is of utmost importance in the clinical management of $\mathrm{CF}^{2 \mathrm{a}}$

Expectorated sputum specimens are a reliable source for identification of lower airway bacteria, but very few children aged less than 10 years can expectorate effectively. Bronchoalveolar lavage (BAL) may be superior, ${ }^{13}$ but the risk and cost of repeated bronchoscopy and BAL procedures in infants must be taken into consideration when recommending these procedures for routine screening purposes. Cough swabs have been used in these patients as a substitute source for isolating bacterial pathogens. However, studies have shown that although cough swabs have a high positive predictive value, a negative cough swab does not rule out the presence of pathogenic microorganisms in the lower airways of patients with $\mathrm{CF}^{4}{ }^{4}$

The aim of this study was to look at the cough plate as an alternative method of obtaining samples in CF patients. Historically cough plates were used in diagnosis of whooping cough but have been superseded by the use of per nasal swab. ${ }^{56}$ In order to determine the diagnostic accuracy of the cough plate we compared cough plates with dry cough swabs, moistened cough swabs, and spontaneously expectorated sputum samples in CF patients attending the outpatient clinic, using sputum as the "gold standard".

\section{METHODS}

A randomised, prospective study of respiratory sample collection was conducted between April and December 2001 in CF patients aged between 8 and 16 years, expectorating sputum and attending the tertiary CF clinic at Birmingham Children's Hospital.

Four different specimens (sputum, cough plate (CP; 5\% horse blood agar), dry swab (DS), and moistened cough swab (MS)) were collected from each patient. It was felt that moistening swabs with sterile saline might enhance microbial adherence to or survival on swabs. Thirty one patients in all who could produce sputum were included in the study. Patients were asked to cough twice each onto cough plate, dry and moistened cough swabs, and to produce a sample of sputum before physiotherapy. All specimens were taken to the laboratory and processed immediately to optimise isolation rates. Cough swabs and sputum were inoculated onto 5\% blood agar, chocolate agar (for isolation of Haemophilus influenzae), cepacia selective agar (for Burkholderia cepacia) and ALB agar (for fungi), as per routine laboratory procedure for specimens from CF patients. All specimens were cultured at $37^{\circ} \mathrm{C}$ with $5 \%$ carbon dioxide for 48 hours. Antibiotics were commenced empirically at the clinic visit according to respiratory symptoms, but treatment was altered as directed by the result of the microbiological culture.

Ethics approval for this study was obtained from the Birmingham Children's Hospital Research Group.

Statistical advice was sought from Dr Paul Davies, University of Birmingham. Patients were to be allocated at random to an order of obtaining the four samples such that each of the 24 possible orders was used twice, thus balancing out possible method order effects on the results. However, the study terminated after 31 patients when the second cycle of 24 was only partially done. The study is therefore almost but not totally balanced with respect to order effects. Twenty of 31 had positive sputum culture. The results of the other three methods in these 20 patients were compared using the McNemar test for paired responses.

\section{RESULTS}

Of 31 patients, clinically significant microorganisms were cultured on sputum specimens in 20 patients (table 1 ). Clinically significant microorganisms were not isolated in the remaining 11 patients by any method. Some of the sputum cultures grew more than one species and similarly more than one species was isolated on one cough swab. None of the cough plates isolated more than one microorganism, but Pseudomonas aeruginosa was isolated on one cough plate, which had not grown on corresponding sputum culture. 
Table 1 Microorganisms isolated by the four methods

\begin{tabular}{lllllll}
\hline & $\begin{array}{l}\text { Pseudomonas } \\
\text { aeruginosa }\end{array}$ & $\begin{array}{l}\text { Candida } \\
\text { albicans }\end{array}$ & $\begin{array}{l}\text { Staphylocccus } \\
\text { aureus }\end{array}$ & MRSA $^{*}$ & $\begin{array}{l}\text { Aspergillus } \\
\text { spp. }\end{array}$ & Escherichia coli \\
\hline Sputum & 9 & 5 & 3 & 2 & 4 & 1 \\
Cough plate & 10 & 0 & 3 & 3 & 0 & 0 \\
$\begin{array}{l}\text { Dry swab } \\
\text { Moistened swab }\end{array}$ & 5 & 1 & 1 & 1 & & 0 \\
\hline *MRSA, methicillin resistant Staphylococcus aureus.
\end{tabular}

Of 20 patients who had a positive sputum culture, 16 had a positive culture on cough plate (sensitivity $80 \%$, 95\% CI $56-$ $94 \%$ ), seven had a positive culture on moistened swab (sensitivity 35\%, CI 95\% 15-59\%), and seven had a positive culture on dry swab (sensitivity 35\%, CI 95\% $15-59 \%$ ) (table 2).

When comparing cough plates with either moistened or dry swabs, the cough plate method found significantly more positive cases than either of the cough swabs $(p=0.012$ for dry swab and moistened swab, both using the McNemar test).

When asked, all 31 patients reported that they preferred the cough plate method to cough swabs.

\section{DISCUSSION}

The principal findings of this study are firstly that the cough plate was a more sensitive method when compared to cough swabs, for isolating bacteria that can be readily cultured on blood agar. In our study and the Brompton study ${ }^{4}$ cough swabs were taken in an outpatient setting, using a method involving no direct contact with the oropharynx. The Brompton study ${ }^{4}$ showed that cough swabs compared with sputum samples had a specificity of $100 \%$ but sensitivity of $34 \%$. Our study showed a comparable sensitivity of $35 \%$. These results conflict with other investigations ${ }^{13}$ where oropharyngeal (OP) secretions were studied, which showed that OP cultures have poor sensitivity but a high negative predictive value. These OP swabs were obtained by direct contact with the pharynx under general anaesthesia.

Secondly, all the patients preferred cough plates to cough swabs. This is important, as many CF patients dislike cough swabs. It is perceived as intrusive and some patients develop a phobia to cough swabs. Cough swabs have been described as the "worst thing done in the CF clinic". Cough plates have a bigger surface area, are non-intrusive, and patients cooperate better to give a good forceful cough. All of these factors increase the chance of detecting pathogenic microorganisms from the lower respiratory tract in CF patients. There is no additional cost to the department other than the need to ensure prompt delivery of cough plates from department to clinic and back from clinic to the microbiology department.

It is important to note that no fungi were isolated from the cough plates, whereas nine sputum samples yielded Candida or Aspergillus. Although clinically it would be important to exclude the presence of Aspergillus in the respiratory tract of CF patients, the isolation of Candida is less likely to be clinically significant, and more likely to represent oropharyngeal contamination. There are a number of possible explanations for the discrepancy in isolation of fungal species. In this pilot study neither the culture medium used nor the incubation conditions of the cough plates were optimal for the isolation of fungi. In addition cough plate samples may be less prone than cough swabs to contamination by fungi from the oropharynx (especially Candida); this may be an advantage of cough plates. Finally, cough plates may be inherently less sensitive than sputum for isolation of fungi. Further studies will need to clarify the reason for negative fungal cultures on cough plates.

We have subsequently compared cough plates with cough swabs in non-sputum producing patients. The preliminary results in the 24 non-expectorating patients show that nine had a positive culture on cough plate (six Pseudomonas aeruginosa, one Aspergillus spp., one Staphylococcus aureus, one Streptococcus pneumoniae), but all had negative dry and moistened swabs. Given that the Brompton study showed only $8 \%$ frequency in cough swabs in asymptomatic patients, ${ }^{4}$ it is not surprising that a sample of 24 failed to find any positive cases. These interesting results obviously need confirmation, and the study is ongoing.

Historically cough plates were used in the isolation of Bordetella pertussis in whooping cough where a Petri plate containing suitable medium (Bordet-Gengou or substitute) was held in front of the mouth of the coughing patient because there was difficulty in obtaining productive sputum from infants. Although cough plates had an advantage that they did not distress patients, they were subject to excessive contamination. Nasopharyngeal swabs are now preferred to cough plates as they are simpler and help in identifying early atypical cases. ${ }^{56}$

Our study looked at organisms growing on blood agar, but selective culture media are required for isolation of other organisms, for example, $H$ influenzae and $B$ cepacia, as well as fungi. As already discussed the cough plates had no positive fungal cultures in our study. One advantage of cough swabs is that they can be inoculated onto a series of different media. In order to detect the full range of potential pathogens using a cough plate, either a series of cough plates or a single cough

Table 2 Comparison of dry and moistened cough swabs with cough plate cultures in patients with positive sputum culture

\begin{tabular}{|c|c|c|c|c|c|c|}
\hline & \multicolumn{3}{|c|}{ Dry swab } & \multicolumn{3}{|c|}{ Moistened swab } \\
\hline & - & + & Total & - & + & Total \\
\hline Cough plate - & $\begin{array}{l}3 \\
75.0 \%\end{array}$ & $\begin{array}{l}1 \\
25.0 \%\end{array}$ & $\begin{array}{l}4 \\
100.0 \%\end{array}$ & $\begin{array}{l}3 \\
75.0 \%\end{array}$ & $\begin{array}{l}1 \\
25.0 \%\end{array}$ & $\begin{array}{l}6 \\
100.0 \%\end{array}$ \\
\hline Cough plate + & $\begin{array}{l}10 \\
62.5 \%\end{array}$ & $\begin{array}{l}6 \\
37.5 \%\end{array}$ & $\begin{array}{l}16 \\
100.0 \%\end{array}$ & $\begin{array}{l}10 \\
62.5 \%\end{array}$ & $\begin{array}{l}6 \\
37.5 \%\end{array}$ & $\begin{array}{l}16 \\
100.0 \%\end{array}$ \\
\hline Total Count & $\begin{array}{l}13 \\
65.0 \%\end{array}$ & $\begin{array}{l}7 \\
35.0 \%\end{array}$ & $\begin{array}{l}20 \\
100.0 \%\end{array}$ & $\begin{array}{l}13 \\
65.0 \%\end{array}$ & $\begin{array}{l}7 \\
35.0 \%\end{array}$ & $\begin{array}{l}20 \\
100.0 \%\end{array}$ \\
\hline
\end{tabular}


plate containing different media would need to be collected from each patient. Further work is therefore necessary to investigate the optimal use of cough plates.

\section{Conclusion}

Cough plates have the potential to detect more pathogens than cough swabs and hence merit closer observation. Further areas of investigation include: (1) whether we can isolate Pseudomonas or other unusual organisms earlier (using more than one cough plate or cough plate with different media); (2) whether clinically significant microorganisms causing acute exacerbations can be identified more often on cough plates; and (3) whether cough plates detect more pathogens than cough swabs in non-sputum producers. A study looking at role of different media and the isolation of pathogens in non-sputum producers is already underway.

We gratefully acknowledge the help of Dr Noreen West and her preliminary work looking at respiratory samples taken in the CF outpatient setting.

\section{Authors' affiliations}

S Maiya, M Desai, A Baruah, P Weller, J R Clarke, Department of Respiratory Medicine, Birmingham Children's Hospital, Birmingham, UK J Gray, Department of Microbiology, Birmingham Children's Hospital, Birmingham, UK

\section{REFERENCES}

1 Armstrong DS, Grimwood K, Carlin JB, et al. Bronchoalveolar lavage or oropharyngeal cultures to identify lower respiratory pathogens in patients with cystic fibrosis. Pediatr Pulmonol 1996;21:267-75.

2 Hoiby N. Microbiology of cystic fibrosis. In: Hudson M, Geddes DM, eds. Cystic fibrosis. London: Chapman \& Hall Medical, 1995:75-98.

2a Ramsey BW. What is the role of upper airway bacterial cultures in patients with cystic fibrosis? Pediatr Pulmonol 1996;21:265-6.

3 Rosenfeld M, Emerson J, Accurso F, et al. Diagnostic accuracy of oropharyngeal cultures in infants and young children with cystic fibrosis. Pediatr Pulmonol 1999;28:321-8.

4 Equi AC, Pike SE, Davies J, et al. Use of cough swabs in cystic fibrosis clinic. Arch Dis Child 2001;85:438-9.

5 Stokes EJ, Ridgeway GL, Wren MWD. Clinical microbiology, 7th edn. Edward Arnold, 1993:72.

6 Frobisher M (revised by Fuerst R). Frobisher and Fuerst's microbiology in health and disease, 14th edn. New York: WB Saunders \& Co., 1978:456.

\section{IMAGES IN PAEDIATRICS}

\section{Twelfth nerve palsy due to a retropharyngeal tuberculous abscess}

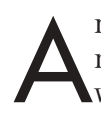
n 11 year old Pakistani boy presented with a four month history of dysphagia and weight loss of $10 \mathrm{~kg}$, which followed a sore throat. Physical findings included diffuse swelling below the angle of the jaw on the left, stiffness of the neck, and wasting of the left side of the tongue with deviation to the left, on protrusion (fig 1). MRI (post-contrast stir sequence) showed an extensive cystic/solid mass in the retropharyngeal and prevertebral spaces, with possible extension to the base of skull (fig 2). Incision and drainage yielded non-offensive pus, which subsequently grew isoniazid resistant Mycobacterium tuberculosis.

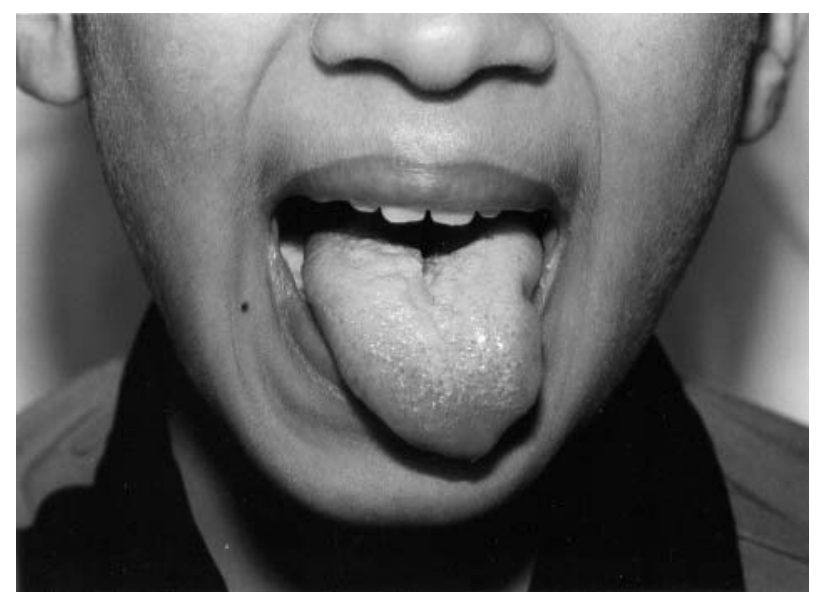

Figure 1 Twelfth nerve palsy resulting in wasting of the left half of tongue with deviation to the left on protrusion.

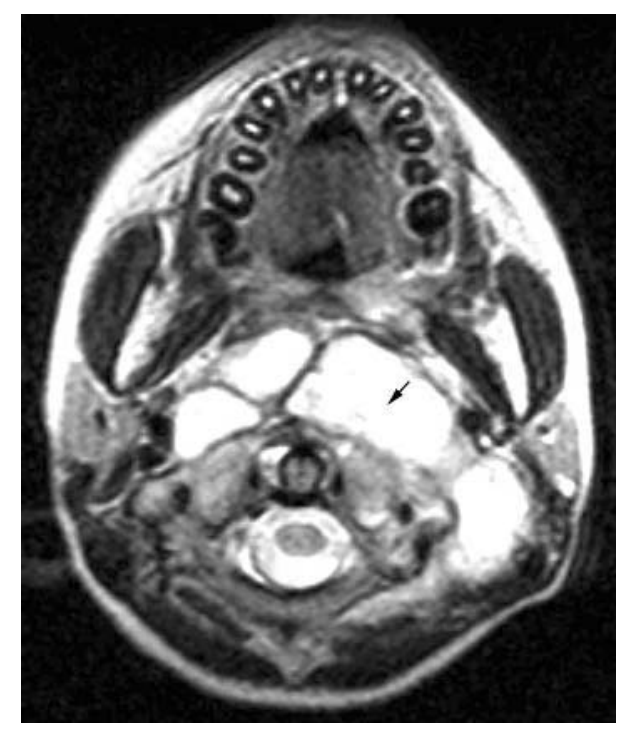

Figure 2 MRI neck/skull base showing partly cystic and partly solid mass occupying the retropharyngeal space and prevertebral space (marked with arrow).

S S Gunawardana

A R Earley

A J Pollard

D Bethell

Wycombe Hospital, High Wycombe, UK; gunawardana@lineone.net 